

О. Е. Орел, Критерий траекторной эквивалентности интегрируемых гамильтоновых систем в окрестности эллиптических орбит. Траекторный инвариант задачи Лагранжа, Матем. сб., 1997, том 188, номер 7, 139-160

DOI: https://doi.org/10.4213/sm249

Использование Общероссийского математического портала Math-Net.Ru подразумевает, что вы прочитали и согласны с пользовательским соглашением

http://www . mathnet.ru/rus/agreement

Параметры загрузки:

IP: 34.239 .49 .27

26 апреля 2023 г., 15:48:27

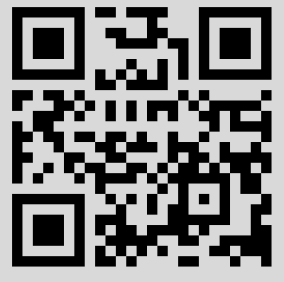


УДК 514.745 .82

\author{
О.Е. Орел
}

\title{
Критерий траекторной эквивалентности интегрируемых гамильтоновых систем в окрестности эллиптических орбит. Траекторный инвариант задачи Лагранжа
}

\begin{abstract}
В работе получен критерий непрерьвной и гладкой траекторной эквивалентности интегрируемых гамильтоновых систем с $n$ степенями свободы в окрестности компактных эллиптических орбит. Кроме того, построен полньй гладкий траекторньй инвариант невырожденной интегрируемой гамильтоновой системы с двумя степенями свободы в окрестности эллиптической особой точки и предложено правило выгисления этого траекторного инварианта. Траекторный инвариант вычислен для интегрируемых систем Лагранжа в динамике твердого тела. Тем самьм в явном виде указано разбиение всех систем Лагранжа на классы траекторно эквивалентных в окрестности положений равновесия.

Библиографиия: 8 названий.
\end{abstract}

\section{§1. Введение}

Пусть $M^{4}$ - симплектическое многообразие, $\mathbf{v}=\operatorname{sgrad} h$ - гамильтонова система на нем, интегрируемая по Лиувиллю при помощи дополнительного интеграла $f$. А. Т. Фоменко и А.В. Болсинов построили в [1] новый топологический инвариант, классифицирующий интегрируемые гамильтоновы системы (ИГС) с двумя степенями свободы на поверхностях постоянной энергии с точностью до траекторной эквивалентности. Напомним, что две гладкие динамические системы на многообразиях $M$ и $M^{\prime}$ называются непрерывно (соответственно, гладко) траекторно әквивалентными, если сушествует гомеоморфизм (соответственно, диффеоморфизм) одного многообразия на другое, переводящий траектории первой системы в траектории второй с сохранением ориентации. При этом преобразование не обязано сохранять время вдоль траектории. В соответствии с теорией траекторной классификации каждой динамической системе, ограниченной на поверхность уровня гамильтониана $Q^{3}=\{h=$ const $\}$, сопоставляется инвариант $W^{* t}\left(Q^{3}\right)$ или $t$-молекула. Две интегрируемые системы траекторно эквивалентны тогда и только тогда, когда их $t$-молекулы совпадают.

Работа выполнена при поддержке Международного научного фонда (грант № а96-45) и INTAS-94-3666. 
Общие идеи и подходы, развитые в [1], использованы в данной работе для траекторной классификации ИГС с произвольньм числом степеней свободы, но рассматриваемых уже не на поверхности уровня гамильтониана, а в малой окрестности эллиптической особенности отображения момента.

Рассмотрим ИГС $\mathbf{v}=\operatorname{sgrad} h$ с $n$ степенями свободы, заданную на некотором симплектическом многообразии $M^{2 n}$. Предположим, что эта система интегрируема по Лиувиллю при помоши интегралов $\mathbf{f}=\left(f_{1}, f_{2}, \ldots, f_{n}\right), f_{1}=h$. Определим пуассоново действие групшы $\mathbb{R}^{n}$ на многообразии $M^{2 n}$ как действие, порожденное сдвигами вдоль интегральных траекторий полей $\operatorname{sgrad} f_{i}, i=1, \ldots, n$. Слоение $M^{2 n}$ на орбиты пуассонова действия называется лиувиллевым слоением. Если в некоторой точке компактной орбиты (а значит, и во всех ее точках) векторные поля $\operatorname{sgrad} f_{i}$ линейно независимы, то по теореме Лиувилля эта орбита диффеоморфна $n$-мерному тору, причем потоки sgrad $f_{i}$ линеаризуются в некоторых координатах, определенных в окрестности тора. Орбиты пуассонова действия $O(x)$, проходящие через точку $x \in M^{2 n}$, где поля $\operatorname{sgrad} f_{i}$ линейно зависимы, называются особыми. Размерность (ранг) особой орбиты совпадает с рангом матрицы $\operatorname{sgrad} \mathbf{f}$ (или, что то же самое, $d \mathbf{f})$.

Пусть даны две ИГС $\mathbf{v}$ и $\mathbf{v}^{\prime}$ на многообразиях $M$ и $M^{\prime}$, соответственно. Рассмотрим особые орбиты пуассонова действия $O\left(x_{0}\right)$ и $O\left(x_{0}^{\prime}\right)$ и связные компоненты множеств $U\left(x_{0}\right)=\mathbf{f}^{-1}\left(\mathbf{f}\left(O\left(x_{0}\right)\right)\right)$ и $U^{\prime}\left(x_{0}^{\prime}\right)=\left(\mathbf{f}^{\prime}\right)^{-1}\left(\mathbf{f}^{\prime}\left(O\left(x_{0}^{\prime}\right)\right)\right)$, содержащие эти орбиты. Интересен вопрос об исследовании траекторной эквивалентности этих систем в окрестностях $U\left(x_{0}\right)$ и $U^{\prime}\left(x_{0}^{\prime}\right)$. В настояшей работе получен критерий траекторной эквивалентности ИГС для случая, когда особая орбита пуассонова действия невырождена и имеет эллиптический тип (определение см. ниже).

ЗАМЕчАНИЕ. В теории динамических систем известен классический результат о траекторной эквивалентности (даже сопряженности) гиперболических векторных полей в окрестности особых точек. Однако этот результат не работает в нашем случае, поскольку ИГС не являются гиперболическими. В частности, в эллиптической особой точке весь спектр векторного поля лежит в точности на мнимой оси.

Рассмотрим особую эллиптическую орбиту пуассонова действия $O$ ранга $k$ (это означает, что $\operatorname{rk} d \mathbf{f}(x)=k$ для всех точек $x \in O$ ). Будем считать гамильтонову систему $\mathbf{v}=\operatorname{sgrad} h$, а также функции $f_{1}, \ldots, f_{n}$ ограниченными на небольшую окрестность $U(O) \subset M^{2 n}$ этой орбиты, не содержашую орбит меньшей размерности. Будем считать функцию $h$ принадлежащей классу гладкости $C^{2}\left(M^{2 n}\right)$, если мы говорим о непрерывном траекторном инварианте, и классу гладкости $C^{4}\left(M^{2 n}\right)$, если мы говорим о гладком инварианте. Будем предполагать, что в окрестности орбиты $O$ все орбиты пуассонова действия компактны. Кроме того, мы будем рассматривать только нерезонансные системы, т.е. будем считать, что торы с иррациональной обмоткой всюду плотны в окрестности $O$. В таком случае лиувиллево слоение не зависит от выбора дополнительных интегралов $f_{2}, \ldots, f_{n}$, а полностью определяется гамильтонианом, поскольку почти все слои могут быть охарактеризованы как замыкания траекторий. Поэтому лиувиллево слоение в данном случае можно определить независимым образом: скажем, что точки $x_{1}, x_{2} \in M^{2 n}$ эквивалентны, если $f\left(x_{1}\right)=f\left(x_{2}\right)$ для любого гладкого 
дополнительного интеграла $f$ гамильтоновой системы. Нетрудно проверить, что это действительно отношение эквивалентности. Тогда в качестве слоения Лиувилля можно взять разбиение $M^{2 n}$ на классы эквивалентности. Слоение Лиувилля окрестности орбиты $O$ (точками которого являются указанные классы эквивалентности) обозначим через $B(U(O))$. Типом точки пространства $B(U(O))$ назовем размерность соответствующего слоя.

Пространство $B(U(O))$ n-мерно и, очевидно, диффеоморфно образу окрестности $U(O)$ при отображении момента $\mathbf{f}: U(O) \rightarrow \mathbb{R}^{n}, \mathbf{f}(x)=\left(f_{1}(x), \ldots, f_{n}(x)\right)$. В свою очередь, в соответствии с теоремой Элиассона [3], образ отображения момента $\mathbf{f}(U(O))$ диффеоморфен окрестности нуля множества $\left\{u_{k+1} \geqslant 0, \ldots, u_{n} \geqslant 0\right\}$ $\subset \mathbb{R}^{n}$. При этом внутренние точки этой области соответствуют неособым $n$-мерным лиувиллевым торам, а граничные - особым орбитам меньшей размерности; точка $(0, \ldots, 0)$ соответствует $k$-мерной эллиптической орбите $O$. Напомним, что образ особых орбит при отображении $\mathbf{f}$ называется бифуркационной диаграммой и обозначается $\Sigma$.

На множестве неособых торов Лиувилля определена непрерывная (гладкая в случае $\left.h \in C^{4}\right)$ проективная функция вращения $\rho$, равная отношению частот квазипериодического движения по неособым торам Лиувилля: $\rho=\left(\alpha^{1}: \cdots: \alpha^{n}\right)$. Из невырожденности орбиты $O$ следует, что координаты функции врашения не могут обращаться в нуль одновременно. Эта функция, однако, определена не однозначно, а с точностью до выбора базиса циклов на орбите $O$. По непрерывности функция $\rho$ продолжается на всю область $U$. Поскольку эта функция зависит только от тора, то ее можно рассматривать как функцию, заданную на лиувиллевом слоении $B(U(O))$.

Теорема. Две нерезонансные ИГС $\mathbf{v} u \mathbf{v}^{\prime}$ с $n$ степенями свободы непрерывно (гладко) траекторно эквивалентны в $2 n$-мерной окрестности әллиптических орбит $O$ и $O^{\prime}$ тогда и только тогда, когда существует гомеоморфизм (диффеоморфизм) $\varphi$ п-мерных лиувиллевых слоений $B(U(O))$ и $B^{\prime}\left(U^{\prime}\left(O^{\prime}\right)\right)$, обладающий следующими свойствами:

1) $\varphi$ сохраняет тип точек $B(U(O))$ и $B^{\prime}\left(U^{\prime}\left(O^{\prime}\right)\right)$,

2) $\varphi$ сохраняет функиию вращения, т.е. $\varphi^{*} \rho^{\prime}=\rho$ при некотором выборе базисов циклов на орбитах $O$ и $O^{\prime}$.

Как уже отмечалось, в нерезонансном случае пространства $B(U(O))$ и $B^{\prime}\left(U^{\prime}\left(O^{\prime}\right)\right)$ определены инвариантно относительно выбора дополнительных интегралов $f_{2}, \ldots, f_{n}$ и, следовательно, сушествование отображения $\varphi$ определяется только гамильтонианом. Однако для практической проверки критерия траекторной эквивалентности удобнее перейти от отображений лиувиллевых слоений к отображениям, заданным на образах отображений моментов.

СлеДСтвИЕ 1. Две нерезонансные ИГС $\mathbf{v} u \mathbf{v}^{\prime}$ с $n$ степенями свободы непрерывно (гладко) траекторно әквивалентны в окрестности әллиптических орбит $O$ и $O^{\prime}$ тогда и только тогда, когда существует гомеоморфизм (диффеоморфизм $\varphi$ некоторой п-мерной окрестности точки $y_{0}=\mathbf{f}(O)$ на 
п-мерную окрестность точки $y_{0}^{\prime}=\mathbf{f}^{\prime}\left(O^{\prime}\right)$, обладающий следующими свойствами:

1) $\varphi$ сохраняет тип точек отображсения момента;

2) $\varphi$ сохраняет функцию вращения, т.е. $\varphi^{*} \rho^{\prime}=\rho$ при некотором выборе базисов ииклов на орбитах $О$ и $O^{\prime}$.

Для невырожденных нерезонансных ИГС с двумя степенями свободы в окрестности эллиптической особой точки (орбиты ранга 0) удается построить полный удобно вычислимый гладкий траекторный инвариант. Условие невырожденности будет сформулировано ниже, отметим только, что почти все ИГС (ограниченные на окрестность эллиптической невырожденной особой точки) являются невырожденными и нерезонансными. В данном случае функция вращения определена однозначно, поскольку базис циклов на 0-мерной орбите нулевой. Кроме того, в данном случае проективную функцию $\rho=\left(\alpha^{1}: \alpha^{2}\right)$ можно представить как скалярную функцию двух аргументов $(h, f)$. Для этого рассмотрим предел функции $\rho$ в точке $y_{0}: \rho_{0}=\left(\alpha_{0}^{1}: \alpha_{0}^{2}\right)$. В силу невырожденности хотя бы одна из частот, скажем, $\alpha^{1}$ отлична от нуля. Тогда положим $\rho=\frac{\alpha^{2}}{\alpha^{1}}-$ скалярная функция. В формулируемом следствии $\rho$ именно такая функция.

СлЕДСТВИЕ 2. Полным гладким траекторным инвариантом невырожденной нерезонансной ИГС с двумя степенями свободы в окрестности эллиптической особой точки $O=x_{0}$ является тройка чисел (последние два определень с точностью до перестановки $) \operatorname{Tr}\left(x_{0}\right)=\left(\rho\left(y_{0}\right), \operatorname{sign}\left(\rho_{1}\left(y_{0}\right)\right), \operatorname{sign}\left(\rho_{2}\left(y_{0}\right)\right)\right)$, где $\rho\left(y_{0}\right)$ - предел функиии вращения в точке $y_{0}$, а $\rho_{i}\left(y_{0}\right)$ - производные функиии $\rho$ в точке у по по направлениям двух касательных $l_{1}$ и $l_{2} \kappa$ бифуркационной диаграмме (рис. 1).

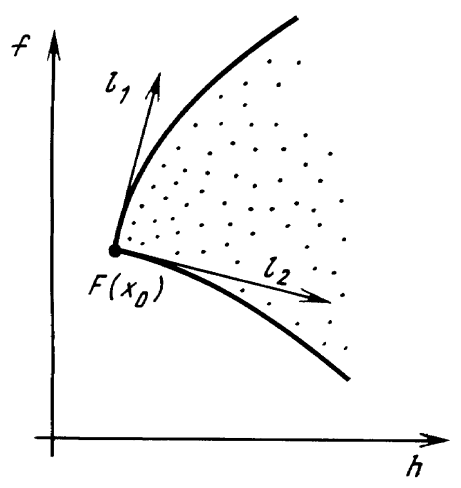

Рис. 1

Это означает, что

1) две невырожденные нерезонансные ИГС с двумя степенями свободы траекторно әквивалентны в окрестностях әллиптических особых точек $x_{0}$ и $x_{0}^{\prime}$ тогда и только тогда, когда тройки $\operatorname{Tr}\left(x_{0}\right)$ u $\operatorname{Tr}\left(x_{0}^{\prime}\right)$ совпадают, 
2) любая тройка $\operatorname{Tr}=\left(\alpha, t_{1}, t_{2}\right), \alpha \in \mathbb{R}, t_{i} \in\{+,-\}$, реализуется как гладкий траекторный инвариант некоторой невырожденной нерезонансной ИГС в окрестности әллиптической особой точки.

Еще раз отметим, что несмотря на присутствие в формулировке конкретного интеграла $f$, от него здесь ничего не зависит, поскольку ни предел функции вращения в особой точке, ни знаки производных не меняются при диффеоморфизмах окрестности образа отображения момента (что как раз и соответствует другому выбору дополнительного интеграла).

В работе также предложено правило вычисления гладкого траекторного инварианта для невырожденных ИГС. Этот инвариант построен для задачи о сферическом маятнике и задачи Лагранжа тяжелого твердого тела.

Волчок Лагранжа - это вращающееся твердое тело, эллипсоид инерции которого является эллипсоидом вращения, а центр тяжести лежит на оси врашения. Задача о движении такого тела трехпараметрическая, параметры содержат информацию о соотношении между моментами инерции тела и коэффициенте линейного потенциала. Хорошо известно, что эта задача полностью интегрируема по Лиувиллю. Динамическая система Лагранжа, описываюшая вращение волчка, зависит от трех параметров, характеризуюших физические свойства волчка. При выполнении некоторого соотношения между параметрами (условия Маевского) вращение волчка с постоянной угловой скоростью вокруг вертикальной оси является невырожденным положением равновесия, или эллиптической особой точкой. В данной работе найден инвариант $\operatorname{Tr}$ этой особой точки в зависимости от параметров системы. Тем самым в явном виде указано разбиение всех систем Лагранжа на классы траекторно эквивалентных в окрестности положений равновесия.

Автор выражает благодарность А.Т. Фоменко и А.В. Болсинову за постановку задачи и постоянное внимание к работе, а также В. В. Козлову за полезные замечания.

\section{§2. Основные определения}

Пусть $x_{0} \in M^{2 n}$ - особая точка отображения момента, $\operatorname{rk} F\left(x_{0}\right)=k$, и пусть $S$ - множество симметричных билинейных форм на $T_{x_{0}} M^{2 n}$. Зададим структуру алгебры Ли на $S$ :

$$
[A, B]_{i j}=A_{i k} \omega^{k l} B_{l j}-B_{i k} \omega^{k l} A_{l j}
$$

где $A=\left(A_{i j}\right) \in S, B=\left(B_{i j}\right) \in S$ и $\omega^{k l} \omega_{l i}=\delta_{i}^{k}, \omega=\left(\omega_{l i}\right)$ - симплектическая структура на $M^{2 n}$. Тогда соответствие $\psi: A \rightarrow A \omega^{-1}$ устанавливает изоморфизм между алгебрами Ли $S$ и $\operatorname{sp}(2 n, \mathbb{R})$. Пусть $\mathbf{g}=\left\{g \mid g=\sum_{i=1}^{n} \lambda_{i} f_{i}, \lambda_{i} \in \mathbb{R}\right.$, $\left.d g\left(x_{0}\right)=0\right\}$, где $f_{i}$ - первые интегралы системы. Тогда $\left[d^{2} g_{\alpha}\left(x_{0}\right), d^{2} g_{\beta}\left(x_{0}\right)\right]=$ $d^{2}\left\{g_{\alpha}, g_{\beta}\right\}\left(x_{0}\right)=0$ при $g_{\alpha}, g_{\beta} \in \mathbf{g}$. Это означает, что подалгебра $K_{\mathbf{g}}=$ $\left\{d^{2} g\left(x_{0}\right) \mid g \in \mathbf{g}\right\} \subset S$ коммутативна.

ОПРЕДЕЛЕНИЕ 2.1. Особая точка $x_{0}$ назЫвается невыро жденной особой точкой отображения момента, если размерность алгебры $K_{\mathbf{g}}$ равна $n-k$. 
ОПРЕДЕЛЕНИЕ 2.2. Эллиптической особой точкой называется невырожденная особая точка, в которой все матрицы подалгебры $\psi\left(K_{\mathbf{g}}\right)$ имеют чисто мнимые собственные значения.

Рассмотрим орбиту $O\left(x_{0}\right)$ пуассонова действия $\Phi: \mathbb{R}^{n} \rightarrow M^{2 n}$, порожденную образами точки $x_{0}$ при сдвигах вдоль траекторий $\operatorname{sgrad} f_{i}, i=1, \ldots, n$.

ОПРЕДЕЛЕНИЕ 2.3. Орбита пуассонова действия, все точки которой невырожденные и эллиптические, назьвается эллиптической орбитой.

ОПРЕДЕЛЕниЕ 2.4. Пусть $\left(s_{i}, \varphi_{i}\right)$ - стандартные переменные “действие-угол" в окрестности некоторого тора Лиувилля $T^{n}$. Согласно теореме Лиувилля на данном торе в координатах $\left(\varphi_{i}\right)$ векторное поле является постоянным. Числом вращения в координатах $\left(\varphi_{i}\right)$ (или в циклах $\gamma_{i}=\left\{\varphi_{j}=\right.$ const, $\left.j \neq i\right\}$ ) назьвается проективный вектор частот квазипериодического движения на лиувиллевом торе:

$$
\rho=\left(\dot{\varphi}_{1}: \dot{\varphi}_{2}: \ldots: \dot{\varphi}_{n}\right) .
$$

Число вращения на торе, очевидно, определено неоднозначно и зависит от выбора базиса $\left(\gamma_{i}\right)$. Однако сушествуют правила, позволяюшие частично избавиться от этой неоднозначности. В нашей задаче в окрестности эллиптической особой точки эти циклы следует выбирать следующим образом:

1) циклы должны непрерывно зависеть от тора;

2) циклы $\gamma_{i}$ должны стягиваться в точку при приближении к $i$-му $(n-1)$-мерному листу бифуркационной диаграммы (образу множества $\left\{u_{i}=0\right\}-$ см. лемму 3.1).

Таким образом, мы однозначно (с точностью до изотопии) определили циклы $\gamma_{i}$, $i=k+1, \ldots, n$. Выбор циклов $\gamma_{i}, i=1, \ldots, k$, неоднозначен, он полностью определяется выбором базиса циклов на орбите $O$.

Рассматривая все семейство неособых торов Лиувилля $\{T\}$ в окрестности эллиптической орбиты, получаем на этом семействе функцию вращения $\rho(T)$. Как будет показано ниже, эта функция непрерывная при $h \in C^{2}$ и гладкая при $h \in C^{4}$. По непрерывности функция $\rho$ может быть продолжена и на особые орбиты пуассонова действия. Поскольку эта функция зависит только от тора, то ее можно рассматривать как функцию, заданную на слоении Лиувилля $B(U(O))$.

\section{§3. Теорема о траекторной классификации}

В этой главе будет доказана следующая

Теорема 3.1. Две нерезонансные ИГС $\mathbf{v} u \mathbf{v}^{\prime}$ с $n$ степенями свободы непрерывно (гладко) траекторно әквивалентны в $2 n$-мерной окрестности эллиптических орбит $O$ и $O^{\prime}$ тогда и только тогда, когда существует гомеоморфизм (диффеоморфизм) $\varphi$ п-мерных лиувиллевых слоений $B(U(O))$ и $B^{\prime}\left(U^{\prime}\left(O^{\prime}\right)\right)$, обладающий следующими свойствами:

1) $\varphi$ сохраняет тип точек $B(U(O))$ и $B^{\prime}\left(U^{\prime}\left(O^{\prime}\right)\right)$,

2) $\varphi$ сохраняет функцию вращения, т.е. $\varphi^{*} \rho^{\prime}=\rho$ при некотором въгборе базисов ииклов на орбитах $О$ и $O^{\prime}$. 
Прежде чем доказывать теорему, заменой координат приведем интегралы системы к "хорошему” виду.

Лемма 3.1. 1) Пусть $\mathbf{v}-$ ИГС с $n$ степенями свободь, $O\left(x_{0}\right)$ - эллиптическая орбита пуассонова действия, $\operatorname{rk} d \mathbf{f}\left(x_{0}\right)=k$. Тогда в некоторой окрестности $U$ орбитьи $O\left(x_{0}\right)\left(U\right.$ гомеоморфна $\left.\mathbb{R}^{n} \times T^{k} \times \mathbb{R}^{n-k}\right)$ существуют симплектические координать $\left(p_{1}, \ldots, p_{n}, q_{1} \bmod 2 \pi, \ldots, q_{k} \bmod 2 \pi, q_{k+1}, \ldots, q_{n}\right)$ такие, что $p_{i}\left(O\left(x_{0}\right)\right)=q_{j}\left(O\left(x_{0}\right)\right)=0$ при $i=1, \ldots, n, j=k+1, \ldots, n$, аинтегралы $f_{i}$ имеют вид

$$
f_{i}=f_{i}\left(u_{1}, \ldots, u_{n}\right) \text {, }
$$

әде $u_{i}=p_{i}$ при $i=1, \ldots, k, u_{i}=p_{i}^{2}+q_{i}^{2}$ при $i=k+1, \ldots, n$. При этом якобиан отобрахсения $\operatorname{det} \partial \mathbf{f} / \partial \mathbf{u}$ отличен от нуля в точке $(0, \ldots, 0)$.

2) Пусть в пространстве $M^{2 n}=\mathbb{R}^{n} \times T^{k} \times \mathbb{R}^{n-k} \quad$ c координатами $\left(p_{1}, \ldots, p_{n}, q_{1} \bmod 2 \pi, \ldots, q_{k} \bmod 2 \pi, q_{k+1}, \ldots, q_{n}\right)$ задана симплектическая структура $\omega=\sum d p_{i} \wedge d q_{i}$. Тогда произвольная гладкая функция $h(\mathbf{u})=h\left(u_{1}, \ldots, u_{n}\right), d h(0, \ldots, 0) \neq 0$, где $u_{i}=p_{i}$ при $i=1, \ldots, k, u_{i}=p_{i}^{2}+q_{i}^{2}$ при $i=k+1, \ldots, n$, задает интегрируемую гамильтонову систему на $M^{2 n}$. Oрбита $(0, \ldots, 0) \times T^{k} \times(0, \ldots, 0)$ будет эллиптической орбитой этой cuстемь.

ДокАЗАтЕльство. 1) Представление (3.1) функций $f_{i}$ в окрестности эллиптической орбиты было получено Элиассоном в [3]. Требование невырожденности особой точки равносильно тому, что якобиан $\operatorname{det} \partial \mathbf{f} / \partial \mathbf{u}$ отличен от нуля в точке $(0, \ldots, 0)$.

2) Очевидно, функции $u_{i}$ суть интегралы системы $\operatorname{sgrad} h$, они почти всюду независимы. Поэтому данная система интегрируема по Лиувиллю. Из условия $d h(0, \ldots, 0) \neq 0$ вытекает, что орбита $(0, \ldots, 0) \times T^{k} \times(0, \ldots, 0)-$ невырожденная эллиптическая орбита этой системы. Лемма доказана.

СЛЕДСТВИЕ 3.1. Множество ростков ИГС в окрестностях әллиптических орбит ранга $k$ находится во взаимно однозначном соответствии с множсеством ростков функиий $h(\mathbf{u})$ от $n$ переменных, заданных в окрестности нуля области $\left\{u_{k+1} \geqslant 0, \ldots, u_{n} \geqslant 0\right\} \subset \mathbb{R}^{n}$, с отличным от нуля дифференииалом $d h(0, \ldots, 0)$.

Описанные в лемме симплектические координаты $\left(p_{i}, q_{i}\right)$ назовем правильными. Для произвольной ИГС рассмотрим правильные координаты в окрестности эллиптической орбиты. Тогда гамильтонова система в новых координатах принимает вид

$$
\begin{array}{lll}
\dot{p}_{i}=0, & \dot{q}_{i}=h_{u_{i}}(\mathbf{u}), & i=1, \ldots, k \\
\dot{p}_{i}=-2 q_{i} h_{u_{i}}(\mathbf{u}), & \dot{q}_{i}=2 p_{i} h_{u_{i}}(\mathbf{u}), & i=k+1, \ldots, n .
\end{array}
$$

Траектории системы находятся в явном виде:

$$
\begin{aligned}
& p_{i}=u_{i}, \quad q_{i}=h_{u_{i}}(\mathbf{u}) t+C_{i} \bmod 2 \pi, \quad i=1, \ldots, k, \\
& p_{i}=\sqrt{u_{i}} \cos \left(2 h_{u_{i}}(\mathbf{u}) t+D_{i}\right), \quad q_{i}=\sqrt{u_{i}} \sin \left(2 h_{u_{i}}(\mathbf{u}) t+D_{i}\right), \quad i=k+1, \ldots, n,
\end{aligned}
$$


где $C_{i}$ и $D_{i}$ - некоторые постоянные, зависящие от начальной точки. Это означает, что движение по тору $\{\mathbf{u}=$ const $\}$ раскладывается в сумму равномерных движений по окружностям $S_{i}=\left\{u_{j}=\mathrm{const}, j \neq i\right\}$ с частотами $h_{u_{i}}(\mathbf{u})$ при $i=1, \ldots, k$ и $2 h_{u_{i}}(\mathbf{u})$ при $i=k+1, \ldots, n$ соответственно. Число врашения на торе равно отношению этих частот:

$$
\rho(\mathbf{u})=h_{u_{1}}(\mathbf{u}): \cdots: h_{u_{k}}(\mathbf{u}): 2 h_{u_{k+1}}(\mathbf{u}): \cdots: 2 h_{u_{n}}(\mathbf{u})
$$

В силу условия $d h(0, \ldots, 0) \neq 0$ все компоненты этого вектора не обращаются в нуль одновременно.

ЗАмЕчАНИЕ. В самом начале мы предположили, что функция $h$ принадлежит классу гладкости $C^{2}$. Это означает, что она принадлежит классу гладкости $C^{1}$ по переменньм $u_{i}$, а функция врашения принадлежит классу $C^{0}$. Если же функция $h$ принадлежит классу гладкости $C^{4}$, то функция вращения оказывается $C^{1}$-гладкой. Только в последнем случае имеет смысл говорить о гладкой эквивалентности.

ДОКАЗАТЕЛЬСТВо ТЕОРЕМЫ. В одну сторону доказательство очевидно: траекторный гомеоморфизм (диффеоморфизм) ИГС в окрестностях эллиптических орбит индуцирует гомеоморфизм (диффеоморфизм) лиувиллевых слоений, сохраняющий функцию врашения.

Докажем обратное утверждение. Пусть $\mathbf{v}=\operatorname{sgrad} h$ и $\mathbf{v}^{\prime}=\operatorname{sgrad} h^{\prime}-$ две нерезонансные ИГС. Ограничим каждую из них на окрестности $U(O)$ и $U^{\prime}\left(O^{\prime}\right)$ соответствующих эллиптических орбит $O$ и $O^{\prime}$. Пусть сушествует указанный в условии гомеоморфизм (диффеоморфизм) лиувиллевых слоений $B(U(O))$ и $B^{\prime}\left(U^{\prime}\left(O^{\prime}\right)\right)$. Докажем, что этот гомеоморфизм (диффеоморфизм) продолжается до траекторного гомеоморфизма (диффеоморфизма) окрестностей $U(O)$ и $U^{\prime}\left(O^{\prime}\right)$. Для этого в обеих системах перейдем к правильным координатам $\left(p_{i}, q_{i}\right)$ и $\left(p_{i}^{\prime}, q_{i}^{\prime}\right)$. Как и раньше, обозначим $u_{i}=p_{i}, u_{i}^{\prime}=p_{i}^{\prime}$ при $i=1, \ldots, k, u_{i}=p_{i}^{2}+q_{i}^{2}, u_{i}^{\prime}=\left(p_{i}^{\prime}\right)^{2}+\left(q_{i}^{\prime}\right)^{2}$ при $i=k+1, \ldots, n$. Из леммы 3.1 следует, что лиувиллевы слоения $B(U(O))$ и $B^{\prime}\left(U^{\prime}\left(O^{\prime}\right)\right)$ диффеоморфны соответственно окрестностям нулей областей $W=$ $\left\{u_{i} \geqslant 0, i=k+1, \ldots, n\right\} \subset \mathbb{R}^{n}$ и $W^{\prime}=\left\{u_{i}^{\prime} \geqslant 0, i=k+1, \ldots, n\right\} \subset \mathbb{R}^{n}$. Тогда гомеоморфизм (диффеоморфизм) $\varphi$ индуцирует гомеоморфизм (диффеоморфизм) областей $W$ и $W^{\prime}$, которьй мы обозначим той же буквой $\varphi$.

Будем считать, что при гомеоморфизме $\varphi$ гиперплоскости $\left\{u_{i}=0\right\}$ переходят в соответствующие гиперплоскости $\left\{u_{i}^{\prime}=0\right\}$ при $i=k+1, \ldots, n$. В противном случае нужно перенумеровать координаты. Пусть $\mathbf{u}^{\prime}=\varphi(\mathbf{u})$. Построим следующее отображение окрестностей $U(O)$ и $U^{\prime}\left(O^{\prime}\right)$ :

$$
\begin{array}{ll}
p_{i}^{\prime}=u_{i}^{\prime}, \quad q_{i}^{\prime}=q_{i}, \quad i=1, \ldots, k, \\
p_{i}^{\prime}=p_{i} \sqrt{\frac{u_{i}^{\prime}}{u_{i}}}, \quad q_{i}^{\prime}=q_{i} \sqrt{\frac{u_{i}^{\prime}}{u_{i}}}, \quad i=k+1, \ldots, n .
\end{array}
$$

Очевидно, что такое отображение взаимно однозначно. Покажем, что оно дает траекторный гомеоморфизм (диффеоморфизм). Действительно, сделаем замену 
времени:

$$
t^{\prime}=\frac{h_{u_{i}}(\mathbf{u})}{h_{u_{i}^{\prime}}^{\prime}\left(\mathbf{u}^{\prime}\right)} t
$$

Здесь коэффициент при $t$ не зависит от номера $i$, так как проективные векторы врашения совпадают в данной точке. При такой замене траектории переходят в траектории:

$$
\begin{aligned}
& p_{i}=u_{i} \mapsto p_{i}^{\prime}=u_{i}^{\prime} \\
& q_{i}=h_{u_{i}}(\mathbf{u}) t+C_{i} \bmod 2 \pi \mapsto q_{i}^{\prime}=h_{u_{i}^{\prime}}^{\prime}\left(\mathbf{u}^{\prime}\right) t^{\prime}+C_{i} \bmod 2 \pi, \quad i=1, \ldots, k ; \\
& p_{i}=\sqrt{u_{i}} \cos \left(2 h_{u_{i}}(\mathbf{u}) t+D_{i}\right) \mapsto p_{i}^{\prime}=\sqrt{u_{i}^{\prime}} \cos \left(2 h_{u_{i}^{\prime}}^{\prime}\left(\mathbf{u}^{\prime}\right) t^{\prime}+D_{i}\right), \\
& q_{i}=\sqrt{u_{i}} \sin \left(2 h_{u_{i}}(\mathbf{u}) t+D_{i}\right) \mapsto q_{i}^{\prime}=\sqrt{u_{i}^{\prime}} \sin \left(2 h_{u_{i}^{\prime}}^{\prime}\left(\mathbf{u}^{\prime}\right) t^{\prime}+D_{i}\right), \quad i=k+1, \ldots, n .
\end{aligned}
$$

Непрерьвность замены $\left(p_{i}, q_{i}\right) \mapsto\left(p_{i}^{\prime}, q_{i}^{\prime}\right)$ очевидна. Проверим ее гладкость для гладкого отображения $\varphi$. Легко видеть, что функции $p_{i}^{\prime}$ и $q_{i}^{\prime}$ непрерывно дифференцируемы по всем переменньм $p_{i}$ и $q_{i}$ при $i=1, \ldots, k$. Проверим непрерывность частных производных $p_{i}^{\prime}$ и $q_{i}^{\prime}$ при $i=k+1, \ldots, n$. Для этого нам потребуется следующая

ЛЕмма 3.2. При $i=k+1, \ldots, n$ имеют место равенства $u_{i}^{\prime}=u_{i} \psi_{i}(\mathbf{u})$, где $\psi_{i}(\mathbf{u})$ - функиии, непрерьвные в области $W=\left\{u_{i} \geqslant 0, i=k+1, \ldots, n\right\} u$ непрерывно дифференцируемые в $\dot{W}=\left\{u_{i}>0\right\} \cap W$. При этом $\psi_{i}(0, \ldots, 0) \neq 0$, $u_{i} \frac{\partial \psi_{i}}{\partial u_{j}}$ и $u_{j} \frac{\partial \psi_{i}}{\partial u_{j}}$ стремятся $\kappa$ нулю при $u_{i} \rightarrow 0$ для всех $j=k+1, \ldots, n$.

ДокАЗАтЕЛЬСтво. Положим $\psi_{i}=\frac{u_{i}^{\prime}}{u_{i}}$ в области $\dot{W} . \quad$ Функция $\psi_{i}$ непрерывно дифференцируема в этой области, так как $u_{i}^{\prime}$ непрерывно дифференцируема. По определению производной $\left.\left.\frac{u_{i}^{\prime}}{u_{i}}\right|_{\mathbf{u}} \rightarrow \frac{\partial u_{i}^{\prime}}{\partial u_{i}}\right|_{\mathbf{u}^{0}}$ при $\mathbf{u} \rightarrow \mathbf{u}^{0}=\left(u_{1}^{0}, \ldots, u_{i-1}^{0}, 0, u_{i+1}^{0}, \ldots, u_{n}^{0}\right)$. Следовательно, функция $\psi_{i}$ продолжается по непрерьвности во всю область $W$. По условию отображение $\varphi$ является диффеоморфизмом в окрестности нуля. Поскольку гиперплоскость $\left\{u_{i}=0\right\}$ переходит в гиперплоскость $\left\{u_{i}^{\prime}=0\right\}$ при $i=k+1, \ldots, n$, то якобиан отображения $\varphi$ в нуле имеет вид

$$
D \varphi=\left(\begin{array}{cc}
* & * * \\
0 & A
\end{array}\right)
$$

где $*$ и $* *$ - некоторые $k \times k$ - и $k \times(n-k)$-матрицы соответственно, 0 - нулевая $(n-k) \times k$-матрица, а $A$ - диагональная матрица с элементами $\left(\psi_{i}(0, \ldots, 0)\right)$ на диагонали. Следовательно, $\left(\psi_{i}(0, \ldots, 0)\right) \neq 0$.

Чтобы доказать последнее утверждение, продифференцируем $u_{i}^{\prime}$ по $u_{i}$ :

$$
\left.\frac{\partial u_{i}^{\prime}}{\partial u_{i}}\right|_{\mathbf{u}}=\psi_{i}(\mathbf{u})+u_{i} \frac{\partial \psi_{i}(\mathbf{u})}{\partial u_{i}}
$$


Так как $\left.\left.\frac{u_{i}^{\prime}}{u_{i}}\right|_{\mathbf{u}} \rightarrow \frac{\partial u_{i}^{\prime}}{\partial u_{i}}\right|_{\mathbf{u}^{0}}$ при $\mathbf{u} \rightarrow \mathbf{u}^{0}=\left(u_{1}^{0}, \ldots, u_{i-1}^{0}, 0, u_{i+1}^{0}, \ldots, u_{n}^{0}\right)$, то $u_{i} \frac{\partial \psi_{i}}{\partial u_{i}} \rightarrow 0$ при $u_{i} \rightarrow 0$. Условия $u_{i} \frac{\partial \psi_{i}}{\partial u_{j}}$ и $u_{j} \frac{\partial \psi_{i}}{\partial u_{j}} \rightarrow 0$ при $u_{i} \rightarrow 0$ для $j \neq i$ следует из того, что $\frac{\partial u_{i}^{\prime}}{\partial u_{j}}$ при $j \neq i$ обрашается в нуль на гиперплоскости $\left\{u_{i}=0\right\}$. Лемма доказана.

Продолжим доказательство теоремы. Рассмотрим, для определенности, функцию $p_{i}^{\prime}=p_{i} \sqrt{\frac{u_{i}^{\prime}}{u_{i}}}=p_{i} \sqrt{\psi_{i}}, i>k$. В соответствии с леммой 3.2 она непрерывна и имеет непрерывные частные производные по всем переменным всюду за исключением, быть может, гиперплоскости $\left\{u_{i}=0\right\}$. Найдем пределы частных производных $p_{i}^{\prime}$ по всем переменным на этой гиперплоскости:

$$
\frac{\partial p_{i}^{\prime}}{\partial p_{i}}=\sqrt{\psi_{i}}+\frac{p_{i}^{2}\left(\partial \psi_{i}\right) /\left(\partial u_{i}\right)}{\sqrt{\psi_{i}}}
$$

Поскольку $u_{i} \frac{\partial \psi_{i}}{\partial u_{i}} \rightarrow 0$ при $u_{i} \rightarrow 0$ и $\psi_{i}(0, \ldots, 0) \neq 0$, то второе слагаемое стремится к нулю при подходе к гиперплоскости, а первое непрерывно. Аналогично,

$$
\begin{aligned}
& \left|\frac{\partial p_{i}^{\prime}}{\partial q_{i}}\right|=\left|\frac{p_{i} q_{i}\left(\partial \psi_{i}\right) /\left(\partial u_{i}\right)}{\sqrt{\psi_{i}}}\right| \leqslant \frac{u_{i}\left(\partial \psi_{i}\right) /\left(\partial u_{i}\right)}{2 \sqrt{\psi_{i}}} \rightarrow 0, \quad u_{i} \rightarrow 0, \\
& \left|\frac{\partial p_{i}^{\prime}}{\partial p_{j}}\right|=\left|\frac{p_{i} p_{j}\left(\partial \psi_{i}\right) /\left(\partial u_{j}\right)}{\sqrt{\psi_{i}}}\right| \leqslant \frac{\left(u_{i}+u_{j}\right)\left(\partial \psi_{i}\right) /\left(\partial u_{j}\right)}{2 \sqrt{\psi_{i}}} \rightarrow 0, \quad u_{i} \rightarrow 0, \\
& \left|\frac{\partial p_{i}^{\prime}}{\partial q_{j}}\right|=\left|\frac{p_{i} q_{j}\left(\partial \psi_{i}\right) /\left(\partial u_{j}\right)}{\sqrt{\psi_{i}}}\right| \leqslant \frac{\left(u_{i}+u_{j}\right)\left(\partial \psi_{i}\right) /\left(\partial u_{j}\right)}{2 \sqrt{\psi_{i}}} \rightarrow 0, \quad u_{i} \rightarrow 0 .
\end{aligned}
$$

Итак, все частные производные $p_{i}^{\prime}$ по $p_{i}, q_{i}$ сушествуют и непрерывны в окрестности нуля. Аналогичное утверждение верно для $q_{i}^{\prime}$. Кроме того, матрица Якоби в нуле имеет диагональный вид и все ее диагональные элементы отличны от нуля. Это доказывает, что построенное отображение $\left(p_{i}, q_{i}\right) \mapsto\left(p_{i}^{\prime}, q_{i}^{\prime}\right)$ является диффеоморфизмом. Теорема доказана.

Для практической проверки критерия траекторной эквивалентности удобнее перейти от отображений лиувиллевых слоений к отображениям, заданным на образах отображений моментов.

СлЕДСТвИЕ 3.2. Две нерезонансные ИГС $\mathbf{v} u \mathbf{v}^{\prime}$ с $n$ степенями свободы непрерывно (гладко) траекторно эквивалентны в окрестности әллиптических орбит $O$ и $O^{\prime}$ тогда и только тогда, когда существует гомеоморфизм (диффеоморфизм $\varphi$ некоторой п-мерной окрестности точки $y_{0}=\mathbf{f}(O)$ на n-мерную окрестность точки $y_{0}^{\prime}=\mathbf{f}^{\prime}\left(O^{\prime}\right)$, обладающий следующими свойствами:

1) $\varphi$ сохраняет тип точек отображсения момента;

2) $\varphi$ сохраняет функиию вращения, т.е. $\varphi^{*} \rho^{\prime}=\rho$ при некотором выборе базисов ииклов на орбитах $O$ и $O^{\prime}$. 
Доказанная теорема упрощает задачу классификации ИГС в окрестности положения равновесия, однако в реальных физических задачах проверить сушествование требуемого преобразования, сохраняющего функцию врашения, достаточно сложно. Поэтому возникает желание построить легко вычисляемьй траекторньй инвариант, классифицирующий ИГС в окрестности эллиптической орбиты. Очевидно, предел функции вращения при стремлении к орбите является траекторньм инвариантом, однако он не полон. Удается построить лишш удобно вычислимый полный гладжий траекторный инвариант, причем результат является содержательным лишш в случае ИГС с двумя степенями свободы. Кроме того, наиболее интересной является проблема о сравнении систем (с траекторной точки зрения) в окрестности эллиптической особой точки или в окрестности положения равновесия. Именно этот результат мы и сформулируем.

Итак, рассмотрим нерезонансную ИГС на многообразии $M^{4}$ и ограничим ее на окрестность эллиптической особой точки $x_{0}$ - эллиптической орбиты ранга 0 . Введем правильные координаты $\left(p_{1}, p_{2}, q_{1}, q_{2}\right)$ в окрестности точки $x_{0}$. Пусть, как и ранњше, $u_{i}=p_{i}^{2}+q_{i}^{2}$. Рассмотрим проективный вектор вращения $\rho\left(u_{1}, u_{2}\right)$ в этой окрестности. Пусть, для определенности, $h_{u_{2}}(0,0) \neq 0$. Тогда можно считать, что $\rho$-это просто функция, равная $\frac{h_{u_{1}}}{h_{u_{2}}}$.

ОПРЕДЕЛЕНИЕ 3.1. ИГС называется невыро жденной в эллиптической особой точке, если

$$
\left.\frac{\partial \rho}{\partial u_{1}}\right|_{(0,0)} \neq 0,\left.\quad \frac{\partial \rho}{\partial u_{2}}\right|_{(0,0)} \neq 0
$$

Поясним, почему этот случай является общим. В соответствии со следствием 3.1, множество ростков ИГС в окрестностях эллиптических особых точек находится во взаимно однозначном соответствии с множеством ростков функций $h\left(u_{1}, u_{2}\right)$ от двух переменных, заданных в окрестности нуля, с отличным от нуля дифференциалом $d h(0,0)$. Для произвольной функции $h$ такого вида выпишем явньй вид дифференциала функции врашения:

$$
d \rho\left(u_{1}, u_{2}\right)=\left(\frac{h_{u_{1} u_{1}} h_{u_{2}}-h_{u_{1}} h_{u_{1} u_{2}}}{h_{u_{2}}^{2}}, \frac{h_{u_{1} u_{2}} h_{u_{2}}-h_{u_{1}} h_{u_{2} u_{2}}}{h_{u_{2}}^{2}}\right)
$$

Условия $\left.\frac{\partial \rho}{\partial u_{1}}\right|_{(0,0)} \neq 0,\left.\frac{\partial \rho}{\partial u_{2}}\right|_{(0,0)} \neq 0$ эквивалентны следуюшим:

$$
\left.\frac{h_{u_{1}}}{h_{u_{2}}}\right|_{(0,0)} \neq\left.\frac{h_{u_{1} u_{1}}}{h_{u_{1} u_{2}}}\right|_{(0,0)},\left.\quad \frac{h_{u_{1}}}{h_{u_{2}}}\right|_{(0,0)} \neq\left.\frac{h_{u_{1} u_{2}}}{h_{u_{2} u_{2}}}\right|_{(0,0)} .
$$

Очевидно, что это случай общего положения. Добавим, что все реальные физические задачи (в частности, задачи динамики твердого тела) являются гладкими и почти все они невырожденные. 
СЛЕДСТВИЕ 3.3. Полным гладким траекторным инвариантом невырожденной нерезонансной ИГС с двумя степенями свободи в окрестности әллиптической особой точки $O=x_{0}$ является тройка чисел (последние два определены с точностью до перестановки $) \operatorname{Tr}\left(x_{0}\right)=\left(\rho\left(y_{0}\right), \operatorname{sign}\left(\rho_{1}\left(y_{0}\right)\right), \operatorname{sign}\left(\rho_{2}\left(y_{0}\right)\right)\right)$, где $\rho\left(y_{0}\right)$ - предел функции вращения в точке $y_{0}$, а $\rho_{i}\left(y_{0}\right)-$ производнье функчии $\rho$ в точке $y_{0}$ по направлениям двух касательных $l_{1}$ и $l_{2} \kappa$ бифуркационной диаграмме.

Это означает, что

1) две невырожденные ИГС с двумя степенями свободы траекторно әквивалентны в окрестностях әллиптических особьх точек $x_{0}$ и $x_{0}^{\prime}$ тогда и только тогда, когда тройки $\operatorname{Tr}\left(x_{0}\right)$ и $\operatorname{Tr}\left(x_{0}^{\prime}\right)$ совпадают;

2) любая тройка $\operatorname{Tr}=\left(\alpha, t_{1}, t_{2}\right), \alpha \in \mathbb{R}, t_{i} \in\{+,-\}$, реализуется как гладкий траекторный инвариант некоторой невырожденной ИГС в окрестности әллиптической особой точки.

ДокАЗАТЕЛЬСТво. Пусть даны две ИГС $\mathbf{v}$ и $\mathbf{v}^{\prime}$. Если эти системы гладко траекторно эквивалентны в окрестностях эллиптических особых точек $x_{0}$ и $x_{0}^{\prime}$, то по теореме 3.1 сушествует диффеоморфизм $\varphi$ областей $\left\{u_{1} \geqslant 0, u_{2} \geqslant 0\right\}$ и $\left\{u_{1}^{\prime} \geqslant 0, u_{2}^{\prime} \geqslant 0\right\}$, сохраняюший функцию врашения. Поэтому $\rho(0,0)=\rho^{\prime}(0,0)$, и знаки производных функций врашения по соответствующим координатам совпадают. В частности, если одна из них невырождена в особой точке, то и вторая невырождена. Необходимость доказана.

Докажем достаточность. Пусть у двух ИГС, невырожденных в окрестностях эллиптических особых точек $x_{0}$ и $x_{0}^{\prime}$, совпадают траекторные инварианты $\operatorname{Tr}\left(x_{0}\right)=\operatorname{Tr}\left(x_{0}^{\prime}\right)$. Рассмотрим линейное преобразование $\varphi_{0}$ :

$$
\left(\begin{array}{l}
u_{1}^{\prime} \\
u_{2}^{\prime}
\end{array}\right)=\left(\begin{array}{cc}
\frac{(\partial \rho) /\left(\partial u_{1}\right)(0,0)}{\left(\partial \rho^{\prime}\right) /\left(\partial u_{1}^{\prime}\right)(0,0)} & 0 \\
0 & \frac{(\partial \rho) /\left(\partial u_{2}\right)(0,0)}{\left(\partial \rho^{\prime}\right) /\left(\partial u_{2}^{\prime}\right)(0,0)}
\end{array}\right)\left(\begin{array}{l}
u_{1} \\
u_{2}
\end{array}\right),
$$

переводящее область $\left\{u_{1} \geqslant 0, u_{2} \geqslant 0\right\}$ в область $\left\{u_{1}^{\prime} \geqslant 0, u_{2}^{\prime} \geqslant 0\right\}$. Легко проверить, что $\varphi_{0}^{*} \rho^{\prime}=\rho+o\left(u_{1}, u_{2}\right)$. Корректируя это преобразование $\varphi\left(u_{1}, u_{2}\right)=$ $\varphi_{0}\left(u_{1}, u_{2}\right)+o\left(u_{1}, u_{2}\right)$ в классе $C^{1}$, нетрудно добиться равенства $\varphi_{0}^{*} \rho^{\prime}=\rho$.

Докажем теперь, что любой инвариант $\left(\alpha, t_{1}, t_{2}\right), t_{i} \in\{+,-\}$, реализуется как гладкий траекторный инвариант некоторой невырожденной ИГС в окрестности эллиптической особой точки. Известно (лемма 3.1 ), что произвольная функция $h\left(u_{1}, u_{2}\right), d h(0,0) \neq 0$, задает ИГС в области $\left\{u_{1} \geqslant 0, u_{2} \geqslant 0\right\} \subset \mathbb{R}^{4}$. Требуется подобрать такую функцию $h$, чтобы для функции вращения были выполнены следуюшие соотношения: $\rho(0,0)=\alpha, \operatorname{sign} \frac{\partial \rho}{\partial u_{1}}(0,0)=t_{1}, \operatorname{sign} \frac{\partial \rho}{\partial u_{2}}(0,0)=t_{2}$. Для $\alpha \neq 0$ положим $h=\alpha u_{1}+u_{2}+t_{1} u_{1}^{2}-\alpha t_{2} u_{2}^{2}$. Тогда из формул (3.3) и (3.4) находим: $\rho(0,0)=\alpha, d \rho(0,0)=\left(2 t_{1}, 2 \alpha^{2} t_{2}\right)$. Для $\alpha=0$ полагаем $h=u_{2}+t_{1} u_{1}^{2}+t_{2} u_{1} u_{2}$. Тогда $\rho(0,0)=0, d \rho=\left(2 t_{1}, t_{2}\right)$. Следствие доказано.

В следуюших параграфах предлагается правило вычисления этого инварианта для всех ИГС в окрестности эллиптической особой точки. 


\section{§4. Вычисление гладкого}

траекторного инварианта в простейшем случае.

\section{Траекторный инвариант сферического маятника}

Пусть гладкая интегрируемая гамильтонова система

$$
\dot{p}_{j}=-\frac{\partial h}{\partial q_{j}}, \quad \dot{q}_{j}=\frac{\partial h}{\partial p_{j}}, \quad j=1,2,
$$

задана в окрестности начала координат $x_{0}=(0,0,0,0)$ пространства $\mathbb{R}^{4}$. Гамильтониан $h(p, q) \in C^{4}\left(\mathbb{R}^{4}\right)$ и дополнительный интеграл $f(p, q)$ будем считать известными. Пусть известно также, что точка $x_{0}$ является невырожденной эллиптической особой точкой системы. Предъявим правило, следуя которому можно ответить на вопрос, находится ли эта система в общем положении в точке $x_{0}$, и в случае положительного ответа вычислить для нее траекторный инвариант.

Напомним, что в соответствии с леммой 3.1 сушествуют правильные симплектические координаты $(p, q)$, в которых функции $h$ и $f$ принимают следуюший вид:

$$
\begin{aligned}
& h=\frac{1}{2} \alpha_{1} u+\frac{1}{2} \alpha_{2} v+\frac{1}{4} a_{11} u^{2}+\frac{1}{2} a_{12} u v+\frac{1}{4} a_{22} v^{2}+\cdots, \\
& f=\frac{1}{2} \beta_{1} u+\frac{1}{2} \beta_{2} v+\frac{1}{4} b_{11} u^{2}+\frac{1}{2} b_{12} u v+\frac{1}{4} b_{22} v^{2}+\cdots,
\end{aligned}
$$

где

$$
u=p_{1}^{2}+q_{1}^{2}, \quad v=p_{2}^{2}+q_{2}^{2} .
$$

Из этого представления и из формулы для дифференциала функции вращения видно, что нам требуется найти нормальную форму Биркгофа степени 4 с точностью до членов степени 5. В этом случае для вычисления траекторного инварианта нужно просто воспользоваться правилом Биркгофа [4], [5] приведения гамильтониана к нормальной форме.

ЗАмечАнИЕ. В теореме Биркгофа требуется, чтобы частоты системы $\alpha_{1} \alpha_{2}$ не удовлетворяли ни одному резонансному соотношению порядка 4 и меньше. В противном случае правило перестает работать. В нашем случае ситуация иная. Мы знаем, что система интегрируема, и имеем в своем распоряжении дополнительньй интеграл. Более того, имеет место неравенство $\alpha_{1} \beta_{2}-\alpha_{2} \beta_{1} \neq 0$. Рассматривая линейные комбинации $h$ и $f$, можно построить функцию с любыми частотами. Поэтому в нашей задаче проблемы резонансов не возникает.

Продемонстрируем работу этого правила на примере сферического маятника. Задача о движении сферического маятника в поле силы тяжести является самой простой и в то же время показательной задачей на вычисление траекторного инварианта в эллиптической особой точке, которая совпадает с положением равновесия системы.

Опишем динамику системы. Фазовое пространство системы - кокасательное расслоение к двумерной сфере $T^{*} S^{2}$. Уравнения движения маятника в поле силы тяжести имеют, очевидно, два интеграла - это энергия $h$ и угловой момент $f$. Рассмотрим в $T^{*} S^{2}$ координаты $(x, y)$, где $x$ - координата точки на сфере, а $y$ 
- ее импульс. Эти координаты являются избыточньми, так как удовлетворяют соотношениям $x_{1}^{2}+x_{2}^{2}+x_{3}^{2}=1, y_{1} x_{1}+y_{2} x_{2}+y_{3} x_{3}=0$. Интегралы в этих координатах записываются следующим образом:

$$
\begin{aligned}
h(x, y) & =\frac{1}{2}\left(y_{1}^{2}+y_{2}^{2}+y_{3}^{2}\right)+x_{3}, \\
f(x, y) & =y_{1} x_{2}-y_{2} x_{1} .
\end{aligned}
$$

Бифуркационная диаграмма для этой задачи построена в [6]. Легко проверяется, что точка $(0,0,-1,0,0,0)$ является эллиптической особой точкой этой системы. В окрестности этой точки в качестве локальных координат можно выбрать симплектические координаты $\left(x_{1}, x_{2}, z_{1}, z_{2}\right)$ (координаты-импульсы). В них функция $h$ принимает вид

$$
\begin{aligned}
h & =\frac{1}{2}\left(z_{1}^{2}+z_{2}^{2}\right)-\frac{1}{2}\left(z_{1} x_{1}+z_{2} x_{2}\right)^{2}-\sqrt{1-x_{1}^{2}-x_{2}^{2}} \\
& =-1+\frac{1}{2}\left(z_{1}^{2}+z_{2}^{2}\right)+\frac{1}{2}\left(x_{1}^{2}+x_{2}^{2}\right)-\frac{1}{2}\left(z_{1} x_{1}+z_{2} x_{2}\right)^{2}+\frac{1}{8}\left(x_{1}^{2}+x_{2}^{2}\right)^{2}+\cdots
\end{aligned}
$$

где многоточием обозначены члены более высокого порядка. Отсюда получаем $\alpha_{1}=\alpha_{2}=1, \rho\left(x_{0}\right)=1$.

На первый взгляд кажется, что квадратичная часть гамильтониана уже приведена к нормальному виду. Однако это не так: дополнительный интеграл $f$ не имеет в этих координатах нормальной формы. Это произошло потому, что частоты системы удовлетворяют резонансному соотношению 2-го порядка $\alpha_{1}-\alpha_{2}=0$. Чтобы правильно выбрать координаты, приведем функцию $f$ также к нормальному виду. Получаем замену:

$$
y_{1}=\frac{p_{1}+p_{2}}{\sqrt{2}}, \quad x_{1}=\frac{q_{1}+q_{2}}{\sqrt{2}}, \quad y_{2}=\frac{q_{2}-q_{1}}{\sqrt{2}}, \quad x_{2}=\frac{p_{1}-p_{2}}{\sqrt{2}}
$$

Функции $h$ и $f$ в новых координатах принимают вид

$$
\begin{aligned}
& h=-1+\frac{1}{2}\left(p_{1}^{2}+p_{2}^{2}\right.\left.+q_{1}^{2}+q_{2}^{2}\right)-\frac{1}{2}\left(p_{1} q_{2}+p_{2} q_{1}\right)^{2} \\
&+\frac{1}{32}\left(\left(q_{1}+q_{2}\right)^{2}+\left(p_{1}-p_{2}\right)^{2}\right)^{2}+\cdots \\
& f=\frac{1}{2}\left(p_{1}^{2}+q_{1}^{2}\right)-\frac{1}{2}\left(p_{2}^{2}+q_{2}^{2}\right) .
\end{aligned}
$$

Заметим, что функции $h$ и $f$ не содержат кубической части, т.е. оказываются приведенными к нормальной форме третьего порядка.

Далее, в соответствии с правилом Биркгофа следует привести наши функции к нормальной форме 4-й степени. Однако в нашей задаче этого можно не делать. Из правила Биркгофа следует, что нужные коэффициенты $a_{11}, a_{12}$ и $a_{22}$ можно определить уже сейчас. Для этого перейдем к комплексным координатам: 
$z_{l}=p_{l}+i q_{l}$. Известно (Биркгоф), что коэффициент при $z_{1}^{2} \bar{z}_{1}^{2}$ равен $\frac{1}{4} a_{11}$, коэффициент при $z_{1} \bar{z}_{1} z_{2} \bar{z}_{2}$ равен $\frac{1}{2} a_{12}$, а коэффициент при $z_{2}^{2} \bar{z}_{2}^{2}$ равен $\frac{1}{4} a_{22}$. В нашем случае получаем

$$
a_{11}=a_{22}=\frac{1}{8}, \quad a_{12}=-\frac{1}{4},
$$

откуда, используя формулу (3.4), немедленно находим знаки производной функции $\rho$ по направлениям касательных к бифуркационной диаграмме: $\operatorname{sign}\left(\rho_{1}\right)=-$, $\operatorname{sign}\left(\rho_{2}\right)=+$. Итак, мы доказали следующее

УТВЕРЖДЕНИЕ 4.1. Гладкий траекторный инвариант сферического маятника в особой точке равен $(1,-,+)$.

\section{§5. Правило вычисления гладкого траекторного инварианта в общем случае}

Пусть в пространстве $\mathbb{R}^{4}$ в окрестности начала координат $x_{0}=(0,0,0,0)$ задана произвольная симплектическая структура $\omega=\sum_{i<j} \omega_{i j} d x^{i} \wedge d x^{j}$ и функция $h(x) \in C^{4}\left(\mathbb{R}^{4}\right)$. Предположим, что гамильтонова система

$$
\dot{x}=\operatorname{sgrad} h
$$

интегрируема в этой окрестности при помощи дополнительного интеграла $f$, и точка $x_{0}$ является эллиптической особой точкой системы. Как и раньше, требуется предъявить правило, отвечающее на вопрос, находится ли эта система в общем положении в точке $x_{0}$, и в случае положительного ответа вычисляющее для нее траекторный инвариант.

Мы могли бы попытаться построить сначала симплектические координаты, а затем воспользоваться предыдушей схемой. В некоторых задачах это действительно можно сделать. В общем случае найти симплектические координаты очень непросто. Однако заметим, что нам и не нужны в точности симплектические, а затем правильные координаты. Ведь для вычисления траекторного инварианта нужны только начальные коэффициенты ряда (4.1) при первых и вторых степенях переменных $u$ и $v$. Поэтому достаточно найти лишь приближение правильных координат с точностью до членов второго порядка. Попытаемся реализовать эту идею в виде следуюшего правила.

Шаг 1. Построение первого приближения правильных координат.

УТВЕРЖДЕНИЕ 5.1. Существует единственная с точностью до поворота в плоскостях $\left(s_{i}, t_{i}\right)$ линейная замена координат $\left(x_{1}, x_{2}, x_{3}, x_{4}\right) \rightarrow\left(s_{1}, t_{1}, s_{2}, t_{2}\right)$ такая, что новые координаты являются первым приближсением правильных координат $(p, q)$ :

$$
\begin{aligned}
& p_{i}=s_{i}+\text { члены более високого порядка, } \\
& q_{i}=t_{i}+\text { члены более высокого порядка. }
\end{aligned}
$$


ДокАЗАТЕЛЬСтво. Найдем собственные значения матрицы $A$ линеаризованной системы $\dot{x}=\operatorname{sgrad} h$ и матрицы $B$ линеаризованной системы $\dot{x}=\operatorname{sgrad} f$ и сделаем линейную замену переменных $(x) \rightarrow(\tilde{s}, \tilde{t})$, приводяшую матрицы $A$ и $B$ к виду

$$
A=\left(\begin{array}{cccc}
0 & -\alpha_{1} & 0 & 0 \\
\alpha_{1} & 0 & 0 & 0 \\
0 & 0 & 0 & -\alpha_{2} \\
0 & 0 & \alpha_{2} & 0
\end{array}\right), \quad B=\left(\begin{array}{cccc}
0 & -\beta_{1} & 0 & 0 \\
\beta_{1} & 0 & 0 & 0 \\
0 & 0 & 0 & -\beta_{2} \\
0 & 0 & \beta_{2} & 0
\end{array}\right)
$$

Эта замена определена однозначно с точностью до поворотов и растяжений в плоскостях $\left(\tilde{s}_{i}, \tilde{t}_{i}\right)$. Коэффициенты растяжения легко вычислить, требуя чтобы гамильтониан и дополнительный интеграл в новых координатах $\left(s_{i}, t_{i}\right)$ имели вид

$$
\begin{aligned}
& h(s, t)=\frac{1}{2} \alpha_{1}\left(s_{1}^{2}+t_{1}^{2}\right)+\frac{1}{2} \alpha_{2}\left(s_{2}^{2}+t_{2}^{2}\right)+\cdots, \\
& f(s, t)=\frac{1}{2} \beta_{1}\left(s_{1}^{2}+t_{1}^{2}\right)+\frac{1}{2} \beta_{2}\left(s_{2}^{2}+t_{2}^{2}\right)+\cdots
\end{aligned}
$$

Поскольку правильные координаты $(p, q)$ определены также с точностью до поворота, получаем требуемое утверждение.

Заметим, что симплектическая структура в особой точке $x_{0}$ в новых координатах имеет вид

$$
\omega\left(x_{0}\right)=d s_{1} \wedge d t_{1}+d s_{2} \wedge d t_{2}
$$

Одновременно с первьм приближением мы получили предел функции вращения в особой точке $x_{0}$ :

$$
\rho\left(x_{0}\right)=\frac{\alpha_{1}}{\alpha_{2}}
$$

Далее нам будет удобно работать в комплексных координатах $z_{l}=s_{l}+i t_{l}$. Введем обозначения, удобные для дальнейшего. Пусть построено $k$-е приближение правильных координат, где $k$ равно 1 или 2. Тогда функции $h$ и $f$ с точностью до членов порядка $k+3$ запишем в виде

$$
\begin{aligned}
& h=\frac{1}{2} \alpha_{1} z_{1} \bar{z}_{1}+\frac{1}{2} \alpha_{2} z_{2} \bar{z}_{2}+\sum_{m, n} D_{m n}^{h} z_{1}^{m_{1}} z_{2}^{m_{2}} \bar{z}_{1}^{n_{1}} \bar{z}_{2}^{n_{2}}+\cdots \\
& f=\frac{1}{2} \beta_{1} z_{1} \bar{z}_{1}+\frac{1}{2} \beta_{2} z_{2} \bar{z}_{2}+\sum_{m, n} D_{m n}^{f} z_{1}^{m_{1}} z_{2}^{m_{2}} \bar{z}_{1}^{n_{1}} \bar{z}_{2}^{n_{2}}+\cdots
\end{aligned}
$$

где суммирование ведется по всем $(m, n)$, удовлетворяющим $m_{1}+m_{2}+n_{1}+n_{2}=$ $k+2, D_{m n}^{h}, D_{m n}^{f}-$ некоторые коэффициенты. Симплектическую структуру запи- 
шем в виде

$$
\begin{aligned}
\omega & =\frac{i}{2}\left(1+\sum_{m, n} d_{m n}^{1 \overline{1}} z_{1}^{m_{1}} z_{2}^{m_{2}} \bar{z}_{1}^{n_{1}} \bar{z}_{2}^{n_{2}}\right) d z_{1} \wedge d \bar{z}_{1} \\
& +\frac{i}{2}\left(1+\sum_{m, n} d_{m n}^{2 \overline{2}} z_{1}^{m_{1}} z_{2}^{m_{2}} \bar{z}_{1}^{n_{1}} \bar{z}_{2}^{n_{2}}\right) d z_{2} \wedge d \bar{z}_{2} \\
& +\frac{i}{2} \sum_{m, n} d_{m n}^{12} z_{1}^{m_{1}} z_{2}^{m_{2}} \bar{z}_{1}^{n_{1}} \bar{z}_{2}^{n_{2}} d z_{1} \wedge d z_{2} \\
& +\frac{i}{2} \sum_{m, n} d_{m n}^{1 \overline{2}} z_{1}^{m_{1}} z_{2}^{m_{2}} \bar{z}_{1}^{n_{1}} \bar{z}_{2}^{n_{2}} d z_{1} \wedge d \bar{z}_{2} \\
& +\frac{i}{2} \sum_{m, n} d_{m n}^{2 \overline{1}} z_{1}^{m_{1}} z_{2}^{m_{2}} \bar{z}_{1}^{n_{1}} \bar{z}_{2}^{n_{2}} d z_{2} \wedge d \bar{z}_{1} \\
& +\frac{i}{2} \sum_{m, n} d_{m n}^{\overline{1} \overline{2}} z_{1}^{m_{1}} z_{2}^{m_{2}} \bar{z}_{1}^{n_{1}} \bar{z}_{2}^{n_{2}} d \bar{z}_{1} \wedge d \bar{z}_{2}+\cdots
\end{aligned}
$$

Здесь суммирование ведется по всем $m_{1}+m_{2}+n_{1}+n_{2}=k, d_{m n}$ - некоторые коэффициенты. Заметим, что в силу вешественности формы $\bar{d}_{m n}^{12}=-d_{n m}^{\overline{1} \overline{2}}, \bar{d}_{m n}^{1 \overline{1}_{n}}=d_{n m}^{1 \overline{1}^{k}}$, $\bar{d}_{m n}^{1 \overline{2}}=d_{n m}^{2 \overline{1}}, \bar{d}_{m n}^{2 \overline{2}}=d_{n m}^{2 \overline{2}}$.

Шаг 2. Построение второго приближения правильных координат.

УТВЕРЖДЕНИЕ 5.2. Существует единственная замена координат вида

$$
z_{l}=w_{l}+\sum_{m, n} C_{m n}^{l} w_{1}^{m_{1}} w_{2}^{m_{2}} \bar{w}_{1}^{n_{1}} \bar{w}_{2}^{n_{2}}
$$

такая, что координаты $(\operatorname{Re} w, \operatorname{Im} w)$ являются вторым приближением правильных координат. Суммирование здесь производится по всем $(m, n)$, удовлетворяющим $m_{1}+m_{2}+n_{1}+n_{2}=2$.

ДокАЗАТЕльство. Сделаем указанную в условии леммы замену с некоторьми (пока неизвестными) коэффициентами $C_{m n}^{l}$. Подставив эту замену в выражения для $h$ и $f$, получаем, что коэффициенты квадратичной части остались прежними, а коэффициенты кубической части изменились. Поскольку мы хотим избавиться от кубической части в гамильтониане и дополнительном интеграле, приравняем коэффициенты при кубических членах к нулю. Имеем следующую систему линейных уравнений на коэффициенты $C_{m n}^{l}$ :

$$
\begin{aligned}
& \frac{1}{2} \alpha_{1}\left(\bar{C}_{n_{1}, n_{2}, m_{1}-1, m_{2}}^{1}+C_{m_{1}, m_{2}, n_{1}-1, n_{2}}^{1}\right) \\
& \quad+\frac{1}{2} \alpha_{2}\left(\bar{C}_{n_{1}, n_{2}, m_{1}, m_{2}-1}^{2}+C_{m_{1}, m_{2}, n_{1}, n_{2}-1}^{2}\right)+D_{m n}^{h}=0 \\
& \frac{1}{2} \beta_{1}\left(\bar{C}_{n_{1}, n_{2}, m_{1}-1, m_{2}}^{1}+C_{m_{1}, m_{2}, n_{1}-1, n_{2}}^{1}\right) \\
& \quad+\frac{1}{2} \beta_{2}\left(\bar{C}_{n_{1}, n_{2}, m_{1}, m_{2}-1}^{2}+C_{m_{1}, m_{2}, n_{1}, n_{2}-1}^{2}\right)+D_{m n}^{f}=0
\end{aligned}
$$


соответственно. Члены с отрицательными индексами считаются равными нулю. Аналогично приравниваем к нулю линейные члены в симплектической структуре:

$$
\begin{aligned}
& \left(m_{1}+1\right) C_{m_{1}+1, m_{2}, n_{1}, n_{2}}^{1}+\left(n_{1}+1\right) \bar{C}_{n_{1}+1, n_{2}, m_{1}, m_{2}}^{1}+d_{m n}^{1 \overline{1}}=0 \\
& \left(n_{1}+1\right) C_{m_{1}, m_{2}, n_{1}+1, n_{2}}^{2}-\left(n_{2}+1\right) C_{m_{1}, m_{2}, n_{1}, n_{2}+1}^{1}+d_{m n}^{1 \overline{2}}=0 \\
& \left(m_{2}+1\right) C_{m_{1}, m_{2}+1, n_{1}, n_{2}}^{1}+\left(n_{1}+1\right) \bar{C}_{n_{1}+1, n_{2}, m_{1}, m_{2}}^{2}+d_{m n}^{2 \overline{1}}=0 \\
& \left(m_{2}+1\right) C_{m_{1}, m_{2}+1, n_{1}, n_{2}}^{2}+\left(n_{2}+1\right) \bar{C}_{n_{1}, n_{2}+1, m_{1}, m_{2}}^{2}+d_{m n}^{2 \overline{2}}=0 .
\end{aligned}
$$

Система уравнений (5.4)+(5.5) обязана быть разрешимой, поскольку правильные координаты всегда сушествуют. Чтобы ответить на вопрос, сколько она имеет решений, найдем пространство решений однородной системы. Из системы (5.4) получаем:

$$
\begin{array}{ll}
C_{2000}^{1}+\bar{C}_{1010}^{1}=0, & C_{1100}^{1}+\bar{C}_{1001}^{1}=0 \\
C_{0200}^{2}+\bar{C}_{0101}^{2}=0, & C_{1100}^{2}+\bar{C}_{0110}^{2}=0
\end{array}
$$

а все остальные коэффициенты равны нулю. Подставив эти выражения в систему (5.5), получаем, что и эти коэффициенты также равны нулю. Итак, однородная система уравнений имеет только нулевые решения. Следовательно, решение неоднородной системы единственно. Имея единственность, мы можем утверждать, что с помощью такой замены мы действительно получили второе приближение правильных координат. Утверждение доказано.

IIIаг 3.

УТВЕРЖДЕНИЕ 5.3. Коэффициенты $a_{i j}$ нормальной формы $h$ могут бъть найдень по следующим формулам:

$$
\begin{aligned}
& a_{11}=-\alpha_{1} d_{1010}^{1 \overline{1}}+4 D_{2020}^{h}, \\
& a_{12}=-\alpha_{1} d_{0101}^{1 \overline{1}}-\alpha_{2} d_{1010}^{2 \overline{2}}+2 D_{1111}^{h}, \\
& a_{22}=-\alpha_{2} d_{0101}^{2 \overline{2}}+4 D_{0202}^{h} .
\end{aligned}
$$

ДокАЗАТЕЛьство. Сделаем замену вида (5.3), полагая $m_{1}+m_{2}+n_{1}+n_{2}=3$, и попытаемся избавиться от квадратичных членов в симплектической структуре. Обнулим коэффициенты при $w_{1} \bar{w}_{1}$ и $w_{2} \bar{w}_{2}$ в членах $d w_{1} \wedge d \bar{w}_{1}$ и $d w_{2} \wedge d \bar{w}_{2}$. Получаем:

$$
\begin{array}{ll}
2\left(C_{2010}^{1}+\bar{C}_{2010}^{1}\right)=-d_{1010}^{1 \overline{1}}, & C_{1101}^{1}+\bar{C}_{1101}^{1}=-d_{0101}^{1 \overline{1}}, \\
2\left(C_{0201}^{2}+\bar{C}_{0201}^{2}\right)=-d_{0101}^{2 \overline{2}}, & C_{1110}^{2}+\bar{C}_{1110}^{2}=-d_{1010}^{2 \overline{2}}
\end{array}
$$


Коэффициенты при $w_{1}^{2} \bar{w}_{1}^{2}, w_{1} w_{2} \bar{w}_{1} \bar{w}_{2}$ и $w_{2}^{2} \bar{w}_{2}^{2}$ в гамильтониане после замены приобретают вид

$$
\begin{aligned}
& \frac{1}{2} \alpha_{1}\left(\bar{C}_{2010}^{1}+C_{2010}^{1}\right)+D_{2020}^{h}, \\
& \frac{1}{2} \alpha_{1}\left(\bar{C}_{1101}^{1}+C_{1101}^{1}\right)+\frac{1}{2} \alpha_{2}\left(\bar{C}_{1110}^{2}+C_{1110}^{2}\right)+D_{1111}^{h}, \\
& \frac{1}{2} \alpha_{2}\left(\bar{C}_{0201}^{2}+C_{0201}^{2}\right)+D_{0202}^{h},
\end{aligned}
$$

соответственно. Поскольку искомые числа $\frac{1}{4} a_{11}, \frac{1}{2} a_{12}$ и $\frac{1}{4} a_{22}$ суть коэффициенты при $w_{1}^{2} \bar{w}_{1}^{2}, w_{1} w_{2} \bar{w}_{1} \bar{w}_{2}$ и $w_{2}^{2} \bar{w}_{2}^{2}$ в гамильтониане, утверждение доказано.

\section{§6. Траекторный инвариант интегрируемой задачи Лагранжа}

Рассмотрим задачу о движении тяжелого твердого тела в трехмерном евклидовом пространстве. Напомним, что движение твердого тела описывается системой уравнений

$$
\left\{\begin{array}{l}
\dot{s}=[s, \omega]+[r, u] \\
\dot{r}=[r, \omega]
\end{array}\right.
$$

Здесь $s$ - кинетический момент тела; $\omega$ - угловая скорость тела; $r$ - единичный вектор, направленный по вертикальной оси; $u=m x$, где $m$ - масса тела, а $x=\left(x_{1}, x_{2}, x_{3}\right)$ - радиус-вектор центра масс. Эта система, заданная в пространстве $\mathbb{R}^{6}=\mathbb{R}^{3}(s) \times \mathbb{R}^{3}(r)$, имеет три интеграла

$$
\begin{gathered}
f_{1}=r_{1}^{2}+r_{2}^{2}+r_{3}^{2}, \\
f_{2}=s_{1} r_{1}+s_{2} r_{2}+s_{3} r_{3}, \\
H=\frac{1}{2}\left\langle s, h^{-1}(s)\right\rangle+m\langle x, r\rangle,
\end{gathered}
$$

где $h=\operatorname{diag}(A, B, C), A, B, C$ - главные моменты инерции тела, а через $\langle$,$\rangle обо-$ значено стандартное скалярное произведение в пространстве $\mathbb{R}^{3}(s)$.

Как известно, можно задать симплектическую структуру на совместной поверхности уровня $M^{4}=\left\{f_{1}=1, f_{2}=g\right\}$ через структуру скобок Пуассона

$$
\left\{s_{i}, s_{j}\right\}=\varepsilon_{i j k} s_{k}, \quad\left\{r_{i}, r_{j}\right\}=0, \quad\left\{s_{i}, r_{j}\right\}=\varepsilon_{i j k} r_{k} .
$$

При этом система (6.1) оказывается гамильтоновой на этом многообразии. Случай Лагранжа характеризуется тем, что $A=B>C$ и $x_{1}=x_{2}=0$. Это означает, что эллипсоид инерции твердого тела является эллипсоидом врашения, а центр тяжести лежит на оси врашения. Система (6.1) в случае Лагранжа полностью интегрируема по Лиувиллю на $M^{4}$. После линейной замены координат, сохраняющей пуассонову структуру, гамильтониан и дополнительный интеграл для случая волчка Лагранжа принимают вид ( $\beta=C / A, a$ - некоторый параметр)

$$
\begin{aligned}
H & =\frac{1}{2}\left(s_{1}^{2}+s_{2}^{2}+\frac{s_{3}^{2}}{\beta}\right)+a r_{3}, \\
F & =s_{3},
\end{aligned}
$$


а сама система записывается так:

$$
\begin{array}{ll}
\dot{s}_{1}=s_{2} s_{3}\left(1-\frac{1}{\beta}\right)-a r_{2}, & \dot{r}_{1}=s_{2} r_{3}-\frac{s_{3} r_{2}}{\beta}, \\
\dot{s}_{2}=-s_{1} s_{3}\left(1-\frac{1}{\beta}\right)+a r_{1}, & \dot{r}_{2}=-s_{1} r_{3}+\frac{s_{3} r_{1}}{\beta} \\
\dot{s}_{3}=0, & \dot{r}_{3}=s_{1} r_{2}-s_{2} r_{1} .
\end{array}
$$

Динамика случая Лагранжа хорошо изучена еше в работах классиков; тонкий топологический и траекторный инварианты для этой задачи построены в работах [7], [8]. Хорошо известно, что при $g^{2}-4 a>0$ (условие Маевского) врашение волчка вокруг вертикальной оси является невырожденньм положением равновесия, отвечающим эллиптической особой точке. В системе координат $\{s, r\}$ эта точка имеет координаты $(0,0, g, 0,0,1)$.

Вычислим гладкий траекторный инвариант в окрестности этой точки. Отметим, что в фазовом пространстве $M^{4}$ можно ввести канонические координаты углы Эйлера и соответствующие им импульсы. Однако, в рассматриваемой точке эти координаты вырождаются. Поэтому в малой окрестности этой точки в качестве локальных координат на $M^{4}$ мы вьнуждены взять координаты $\left(s_{1}, s_{2}, r_{1}, r_{2}\right)$. Тогда другие две координаты выражаются через них следующим образом:

$$
r_{3}=\sqrt{1-r_{1}^{2}-r_{2}^{2}}, \quad s_{3}=\frac{g-s_{1} r_{1}-s_{2} r_{2}}{\sqrt{1-r_{1}^{2}-r_{2}^{2}}} .
$$

Вычисляя матрицу линеаризованной системы, находим собственные значения $\pm i \alpha_{1}$ и $\pm i \alpha_{2}$, где

$$
\alpha_{1}=\frac{g+\sqrt{g^{2}-4 a}}{2}-\frac{g}{\beta}, \quad \alpha_{2}=-\frac{g-\sqrt{g^{2}-4 a}}{2}+\frac{g}{\beta} .
$$

С помощью линейной замены

$$
\begin{array}{ll}
s_{1}=\frac{1}{\left(g^{2}-4 a\right)^{1 / 4}}\left(l v_{1}+k u_{2}\right), & r_{1}=\frac{1}{\left(g^{2}-4 a\right)^{1 / 4}}\left(v_{1}+u_{2}\right), \\
s_{2}=\frac{1}{\left(g^{2}-4 a\right)^{1 / 4}}\left(l u_{1}+k v_{2}\right), & r_{2}=\frac{1}{\left(g^{2}-4 a\right)^{1 / 4}}\left(u_{1}+v_{2}\right),
\end{array}
$$

где

$$
k=\frac{g-\sqrt{g^{2}-4 a}}{2}, \quad l=\frac{g+\sqrt{g^{2}-4 a}}{2},
$$

перейдем к первому приближению $\left(u_{1}, u_{2}, v_{1}, v_{2}\right)$ правильных координат. Эти координаты являются, как нетрудно проверить, также и вторым приближением правильных координат. Подставляя эту замену в выражение для функции $H$, находим

$$
\begin{aligned}
& D_{2020}=\frac{k^{2}}{2 \beta\left(g^{2}-4 a\right)}-\frac{a}{8\left(g^{2}-4 a\right)}, \\
& D_{0202}=\frac{l^{2}}{2 \beta\left(g^{2}-4 a\right)}-\frac{a}{8\left(g^{2}-4 a\right)}, \\
& D_{1111}=\frac{g^{2}+4 a}{4 \beta\left(g^{2}-4 a\right)}-\frac{a}{2\left(g^{2}-4 a\right)} .
\end{aligned}
$$


Симплектическая структура дает

$$
d_{1010}^{1 \overline{1}}=\frac{4 l-3 g}{2\left(g^{2}-4 a\right)}, \quad d_{0101}^{1 \overline{1}}=-d_{1010}^{2 \overline{2}}=-\frac{g}{2\left(g^{2}-4 a\right)}, \quad d_{0101}^{2 \overline{2}}=\frac{-4 k+3 g}{2\left(g^{2}-4 a\right)}
$$

Отсюда с помощью утверждения 5.3 находим коэффициенты $a_{i j}$ :

$$
\begin{aligned}
& a_{11}=\frac{(4 l-3 g)(g / \beta-l)+4 k^{2} / \beta-a}{2\left(g^{2}-4 a\right)}, \\
& a_{12}=\frac{(1-2 / \beta) g^{2}+\left(g^{2}+4 a\right) / \beta-2 a}{2\left(g^{2}-4 a\right)}, \\
& a_{22}=\frac{(4 k-3 g)(g / \beta-k)+4 l^{2} / \beta-a}{2\left(g^{2}-4 a\right)} .
\end{aligned}
$$

Таким образом, получаем следующую теорему.

ТЕОРЕМА 6.1. Гладкий траекторный инвариант әллиптической особой точки $(0,0, g, 0,0,1)$ в задаче Лагранжа есть $\left(\rho_{0}, \operatorname{sign}_{1}, \operatorname{sign}_{2}\right)$, әде

$$
\begin{gathered}
\rho_{0}=\frac{\left(g+\sqrt{g^{2}-4 a}\right)-2 g / \beta}{-g+\sqrt{g^{2}-4 a}+2 g / \beta}, \\
\operatorname{sign}_{1}=\operatorname{sign}\left\{\left(\frac{g}{\beta}-k\right)\left[(4 l-3 g)\left(\frac{g}{\beta}-l\right)+\frac{4 k^{2}}{\beta}-a\right]\right. \\
\left.-\left(l-\frac{g}{\beta}\right)\left[\left(1-\frac{2}{\beta}\right) g^{2}+\frac{g^{2}+4 a}{\beta}-2 a\right]\right\}, \\
\operatorname{sign}_{2}=\operatorname{sign}\left\{\left(\frac{g}{\beta}-k\right)\left[\left(1-\frac{2}{\beta}\right) g^{2}+\frac{g^{2}+4 a}{2 \beta}-a\right]\right. \\
\left.-\left(l-\frac{g}{\beta}\right)\left[(4 k-3 g)\left(\frac{g}{\beta}-k\right)+\frac{4 l^{2}}{\beta}-a\right]\right\},
\end{gathered}
$$

где $k$ и $l$ вычисляются по формулам (6.2).

Итак, множество систем Лагранжа, невырожденных в эллиптической особой точке, - это множество $P$ меры 1 в области $D=\left\{0<\beta<1, g^{2}-4 a>0\right\} \subset \mathbb{R}^{3}$. Действительно, вырожденные системы целиком лежат на двух гиперповерхностях $\left\{\operatorname{sign}_{1}=0\right\} \cup\left\{\operatorname{sign}_{2}=0\right\}$ и образуют поэтому множество меры нуль. Кроме того, область $P$ оказывается расслоенной на двумерные поверхности уровня $\{\rho=\mathrm{const}\}$ и рассеченной на четыре области постоянства знаков $\left(\operatorname{sign}_{1}, \operatorname{sign}_{2}\right)$. Тем самьм, все системы Лагранжа разбиваются на классы траекторно эквивалентных систем, представляющие собой двумерные поверхности в $D$ (быть может, с особенностями). 


\section{Список литературы}

1. Болсинов A. B., Фоменко A. T. Траекторная эквивалентность интегрируемых гамильтоновых систем с двумя степенями свободы. Теорема классификации I, II // Матем. сб. 1994. T. 185. № 4, 5. C. 27-80, 27-78.

2. Bolsinov A. V. Methods of calculation of the Fomenko-Zieschang invariant // Adv. Sov. Math. 1991. V. 4. P. 147-184.

3. Eliasson L. H. Normal form for Hamiltonian systems with Poisson commuting integrals elliptic case // Comm. Math. Helv. 1990. V. 65. P. 4-35.

4. Биркгоф Дж. Д. Динамические системы. М.-Л.: Гостехиздат, 1941.

5. Арнольд В. И. Математические методы классической механики. М.: Наука, 1974.

6. Duistermaat J. J. On global action-angle coordinates // Comm. Pure Appl. Math. 1980. V. 33. P. $687-706$.

7. Oshemkov A. A. Fomenko invariants for the main integrable cases of the rigid body motion equation // Adv. Sov. Math. 1991. V. 6. P. 67-146.

8. Орел O. Е., Такахаши III. Траекторная классификация интегрируемых задач Лагранжа и Горячева-Чаплыгина методами компююерного анализа // Матем. сб. 1996. Т. 187. № 1. C. $95-112$.

Московский государственный

Поступила в редакцию

университет им. М.В. Ломоносова

13.02.1997 